\title{
i-Open Sets and Separating Axioms Spaces
}

Amir A. Mohammed

Sabih W. Askandar

Department of Mathematics \College of Education for Pure Sciences University of Mosul

Mosul-Iraq

Received

02/12/2012
Accepted

11/03/2013

\section{المجاميع المفتوحة من النوع-i وفضاءات بديهيات الانفصال

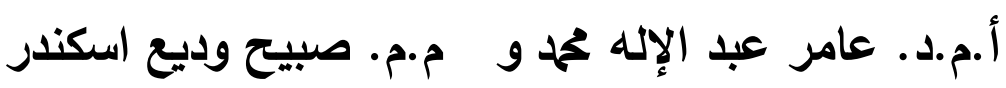 \\ قسم الرياضيات/كلية التربية للعلوم الصرفة/ جامعة الموصل

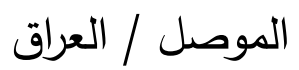

الهدف من هذا البحث هو استخدام نوع من المجاميع المفتوحة المسماة بالمجاميع المفتوحة من النوع-i [9] لدراسة عدة أصناف من فضاءات بديهيات الانفصال للمجاميع المفتوحة، المفتوحة من النوع-o و شبه المفتوحة. فضلا عن ذلك، قمنا بدراسة العلاقة بينها.

$$
\text { الكلمات المفتاحية: }
$$

\section{Abstract:}

The purpose of this paper is using a class of open sets called i-open sets [9] to study some classes of separating axioms spaces for open, $\alpha$-open and semi-open sets. Further, we studied the relations between such spaces. Keywords: $T_{\circ i}, T_{1 i}, T_{2 i}, T_{3 i}, T_{(31 / 2) i}, T_{4 i}, T_{5 i}$.

\section{Introduction:}




\section{i-Open Sets and Separating Axioms Spaces}

Levine in 1963[5], introduced the concept of semi-open sets which improved many important basic theories of the general topology. Njastad in 1965[10], introduced the concept of $\alpha$-open sets which is a subclass of generalized open sets. Also Levine in 1970[6] introduced the concept of generalized closed sets.. Mashhour A.S., Abd El-Monsef M.E. and El-Deeb, S.N., in 1982[8], introduced the concept of Pre-open sets. Dontchev and Maki, in 1999[3], introduced the concept of $\theta$-generalized closed sets. Devi, R., Selvakumar, A. and Parimala, M., in 2011[2], introduced the concept of $\alpha \psi$-closed sets in topological spaces, which, it is complements were called $\alpha \psi$-open sets. Mohammed and Askandar In 2012 [9], introduced the concept of i-open sets which they could to entire them together with many other concepts of Generalized open sets mentioned above. In 2006 Fatima, M. Mohammad introduced Pre- Techonov and PreHausdorff Separation Axioms in Intuitonistic Fuzzy special topological spaces [4] by using the concept of Pre-open sets [8]. In 2011 Y.K. Kim, R. Devi and A. Selvakumar used $\alpha \psi-O p e n$ sets [2] to introduce the concept of Weakly Ultra Separation Axioms [12]. In 2012 Al-Sheikhly, A.H. and Khudhair, H.K.[1] introduced another Type of Separation Axioms Depend on an $\theta g$-open set [3]. The aim of this paper is to introduce another type of Separating Axioms spaces depend on i-open sets [9] for compare with the other separating axioms spaces. This work consists of two sections. In the first one, i-open sets[9] are defined and many related examples have been gave, the comparison between i-open sets, semi-open and $\alpha$-open sets respectively are investigated, New class of mappings named, i-continuous [9] are introduced and comparison among i-continuity [9], continuity [11], semi-continuity [5] and $\alpha$-continuity [13], are investigated (see Corollary 1.28). In the $2^{\text {nd }}$ section, we study many types of separating axioms spaces as like as $\left(T_{\circ}, T_{1}, T_{2}, T_{3}, T_{(31 / 2)}, T_{4}\right.$ and $\left.T_{5}\right)[11],\left(T_{\circ \alpha}, T_{1 \alpha}, T_{2 \alpha}, T_{3 \alpha}, T_{(31 / 2) \alpha}\right.$, $T_{4 \alpha}$ and $\left.T_{5 \alpha}\right),\left(T_{o s}, T_{1 s}, T_{2 s}, T_{3 s}, T_{(31 / 2) s}, T_{4 s}\right.$ and $\left.T_{5 s}\right)$ and $\left(T_{\circ i}, T_{1 i}, T_{2 i}, T_{3 i}\right.$, $T_{(31 / 2) i}, T_{4 i}$ and $\left.T_{5 i}\right)$ by using open, $\alpha$-open[10], semi-open[5] and i-open sets[9] respectively. We give many examples to show that the converse may not be true. Also we discuss the relation among them. (See Corollary 2.5 and Corollaries 2.29). Throughout this work, $(X, \tau)$ and $(Y, \delta)$ are always topological spaces and $f$ is always a mapping $\operatorname{from}(X, \tau)$ into $(Y, \delta)$.

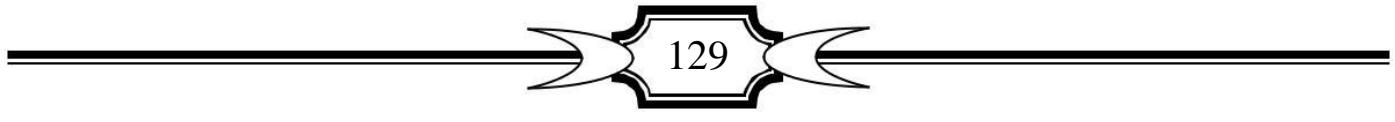




\section{1. i-open sets}

In this Section the concept of i-open sets [9] is defined and their position with the some other classes of generalized-open sets is determined. New class of mappings named i-continuous [9] is introduced and comparison between i-continuity [9], continuity [11], semi-continuity [5] and $\alpha$-continuity [13], are investigated.

Definition1.1. [9] A subset $A$ of $(X, \tau)$ is said to be an i-open if there exists an open set $\mathrm{G} \neq \phi, \mathrm{X}$ such that $A \subseteq C l(A \cap G)$. The complement of an iopen set is called $\mathrm{i}$-closed set.

Example1.2. Let $X=\{a, b, c\}, \tau=\{\phi,\{a\},\{a, c\}, X\}$ by Definition 1.1, iopen sets are: $\phi,\{a\},\{a, c\},\{c\},\{a, b\},\{b, c\}, X$.

Example1.3. Let $X=\{d, e, f\}, \tau=\{\phi,\{d\},\{e\},\{d, e\}, X\}$. Therefore; i-open sets are: $\phi,\{d\},\{e\},\{d, e\},\{d, f\},\{e, f\}, X$.

Theorem1.4. [9] Every open set in a topological space is i-open, but the converse is not true.

Example1.5. Let $X=\{g, h, i\}, \tau=\{\phi,\{g\},\{g, i\}, X\}, A=\{g, h\} . A=\{g, h\}$ is $\mathrm{i}$-open set but it is not open.

Corollary1.6. [9] Every closed set in topological space is i-closed.

Theorem1.7. [9] Every semi-open set in a topological space is i-open.

Example1.8. Let $X=\{j, k, l\}, \tau=\{\phi,\{j, k\}, X\}, A=\{j, l\}$ is i-open set but is not semi-open in $(X, \tau)$.

Corollary1.9. [9] Every $\alpha$-open set in a topological space is i-open.

The converse of Corollary 1.9 is not true. Indeed, In Example 1.8 we see that $A=\{a, c\}$ is $\mathrm{i}$-open set but is not $\alpha$-open $[A \not \subset \operatorname{Int}(C l(\operatorname{Int}(A)))]$.

Corollary1.10. [9] By theorem (1.4), theorem (1.7) and corollary (1.9) we have the following Diagram.
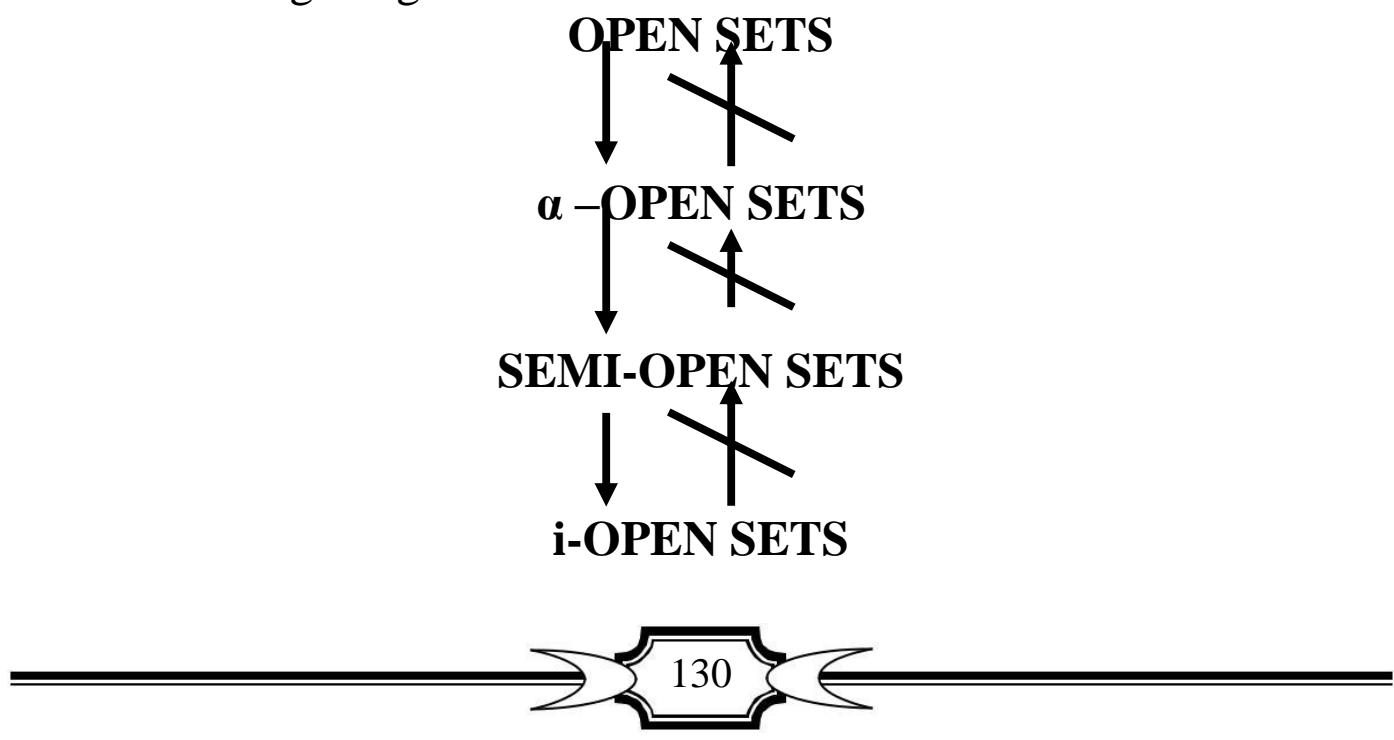


\section{i-Open Sets and Separating Axioms Spaces}

Definition1.11. [9] the extension $\tau^{\mathrm{i}}$ is the family of all i-open subsets of space $X$.

Definition1.12. Let $\left(X, \tau^{i}\right)$ be a topological space and let $A$ be a subset of $\mathrm{X}$ then,

1. The intersection of all $\mathrm{i}$-closed sets containing $A$ is called $\mathrm{i}$-closure of $A$ [9], denoted by $C l_{i}(A): C l_{i}(A)=\bigcap_{i \in A} F_{i} . A \subseteq F_{i} \forall i$ Where, $F_{i}$ is i-closed set $\forall \boldsymbol{i}$ in $\left(X, \tau^{i}\right) . C l_{i}(A)$ is the smallest i-closed set containing $A$.

2. The union of all i-open sets contained in $A$ is called i-Interior of $A$ [9], denoted by $\operatorname{Int}_{\mathrm{i}}(A)$. $\operatorname{Int}_{i}(A)=\bigcup_{i \in A} I_{i}, I_{i} \subseteq A \forall i$, where $I_{\mathrm{i}}$ is an i-open set $\forall i$ in $\left(X, \tau^{i}\right)$. $\operatorname{Int}_{i}(A)$ is the largest $\mathrm{i}$-open set contained in $A$.

Definition1.13. A mapping $f:(X, \tau) \rightarrow(Y, \delta)$ is said to be i-continuous [9](respectively semi-continuous[5]) at the point $x_{o} \in X$ if and only if for each open set $\mathrm{I}^{*} \operatorname{in}(Y, \delta)$ containing $f\left(x_{o}\right)$ there exists an i-open set(respectively semi-open set[5]) $\mathrm{I}$ in $(X, \tau)$ containing $\boldsymbol{x}$ 。 such that $f(\mathrm{I})$ $\subseteq \mathrm{I}^{*} . f$ is $\mathrm{i}$-continuous (respectively semi-continuous) map if it is icontinuous (respectively semi-continuous) at all points of $X$.

Theorem1.14. [9] A mapping $f:(X, \tau) \rightarrow(Y, \delta)$ is i-continuous if and only if,

1. $f^{-1}\left(I^{*}\right)$ is i-open set in $(X, \tau)$ for every open set $\mathrm{I}^{*}$ in $(Y, \delta)$.

2. $f^{-1}\left(\mathrm{I}^{*}\right)$ is i-closed set in $(X, \tau)$ for every closed set $\mathrm{I}^{*}$ in $(Y, \delta)$.

Theorem1.15. [9] Every continuous mapping is i-continuous.

Theorem1.16. [9] Every semi-continuous mapping is i-continuous.

Definition1.17. [9] [13] A mapping $f:(X, \tau) \rightarrow(Y, \delta)$ is said to be $\alpha$ continuous at the point $x_{o} \in X$ if and only if for each open set $I^{*}$ in $(Y, \delta)$ containing $f\left(x_{o}\right)$ there exist an $\alpha$-open set I in $(X, \tau)$ containing $\boldsymbol{x}_{\mathrm{o}}$ such that $f(I) \subseteq I^{*} . f$ is $\alpha$-continuous map if it is $\alpha$-continuous at all points of $X$.

Theorem1.18. [9] [13] A mapping $f$ is $\alpha$-continuous if and only if $f^{-1}\left(I^{*}\right)$ is $\alpha$-open set in $(X, \tau)$ for every open set $I^{*}$ in $(Y, \delta)$.

Theorem1.19. [9] Every $\alpha$-continuous mapping is i-continuous.

Corollary 1.20. [9] the following diagram is true: 


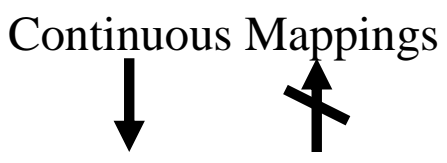

$\alpha$-Continuous Mappings

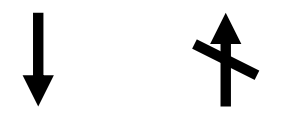

Semi-Continuous Mappings

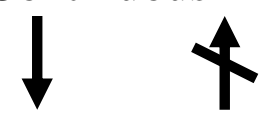

i- Continuous Mappings

\section{2. i-Open Sets and Separating Axioms Spaces}

In this section, we study new types of separating axioms spaces for $\mathrm{i}$ open, semi-open and $\alpha$-open sets for compare and find many relations among them.

Definition2.1. A topological space $(X, \tau)$ is said to be $T_{\text {。 }}$ space [11] (respect. $T_{\circ \alpha}, T_{\text {os }}[7] \operatorname{and} T_{\circ}$ space) if it satisfies Klomogorov axiom[11] (respect. $\alpha$-Klomogorov, s-Klomogorov [7] and i-Klomogorov axiom): $\left[T_{\circ}\left(\right.\right.$ respect. $T_{\circ \alpha}, T_{\circ s}$ and $\left.\left.T_{\circ i}\right)\right]$

$\forall x, y \in X(x \neq y) \exists I \in \tau\left(\right.$ respect. $\tau^{\alpha}, \tau^{s}$ and $\left.\tau^{i}\right)$ s.t. $x \in I, y \notin I$.

Example2.2. Let $X=\{a, b\}, \tau=\{\phi,\{a\}, X\}, \tau^{\alpha}=\tau^{s}=\tau^{i}=\tau,(X, \tau),\left(X, \tau^{\alpha}\right)$ ,$\left(X, \tau^{s}\right)$ and $\left(X, \tau^{i}\right)$ are topological spaces. $a, b \in X(a \neq b) \quad \exists\{a\} \in \tau\left(\right.$ respect. $\tau^{\alpha}, \tau^{s}$ and $\left.\tau^{i}\right)$ s.t $a \in\{a\}, b \notin\{a\}$. Therefore; $(X, \tau)$ is $T_{\circ}, T_{\circ \alpha}, T_{\circ s}$ and $T_{\text {o }}$ space.

Definition2.3. A topological space $(X, \tau)$ is said to be $T_{1}$ space [11] (respect. $T_{1 \alpha}, T_{1 s}[7], T_{1 i}$ space) if it satisfies Frechet axiom [11] (respect. $\alpha$ Frechet, s- Frechet [7] and i-Frechet axiom) :[ $T_{1}\left(\right.$ respect. $\left.\left.T_{1 \alpha}, T_{1 s}, T_{1 i}\right)\right]$ $\forall x, y \in X(x \neq y) \exists I_{1}, I_{2} \in \tau\left(\right.$ respect. $\left.\tau^{\alpha}, \tau^{s}, \tau^{i}\right)$ s.t. $x \in I_{1}, y \notin I_{1} y \in I_{2}, x \notin I_{2}$.

Example2.4. Let $X=\{a, b, c\}, \tau=\{\phi,\{a\},\{b\},\{c\},\{a, b\},\{a, c\},\{b, c\}, X\}$ $, \tau^{\alpha}=\tau^{s}=\tau^{i}=\tau,(X, \tau),\left(X, \tau^{\alpha}\right),\left(X, \tau^{s}\right)$ and $\left(X, \tau^{i}\right)$ are topological spaces. $a, b \in X(a \neq b) \exists\{a\},\{b\} \in \tau, \tau^{\alpha}, \tau^{s}, \tau^{i}$, s.t. $a \in\{a\}, b \notin\{a\} b \in\{b\}, a \notin\{b\}$. 
$a, c \in X(a \neq c) \exists\{a\},\{c\} \in \tau, \tau^{\alpha}, \tau^{s}, \tau^{i}$

s.t. $a \in\{a\}, c \notin\{a\}, c \in\{c\}, a \notin\{c\}$

$b, c \in X(b \neq c) \exists\{b\},\{c\} \in \tau, \tau^{\alpha}, \tau^{s}, \tau^{i}$

s.t. $b \in\{b\}, c \notin\{b\}, c \in\{c\}, b \notin\{c\}$.

Therefore; $(X, \tau)$ is $T_{1}, T_{1 \alpha}, T_{1 s}$, and $T_{1 i}$-space.

Corollary2.5. The following diagram is true.

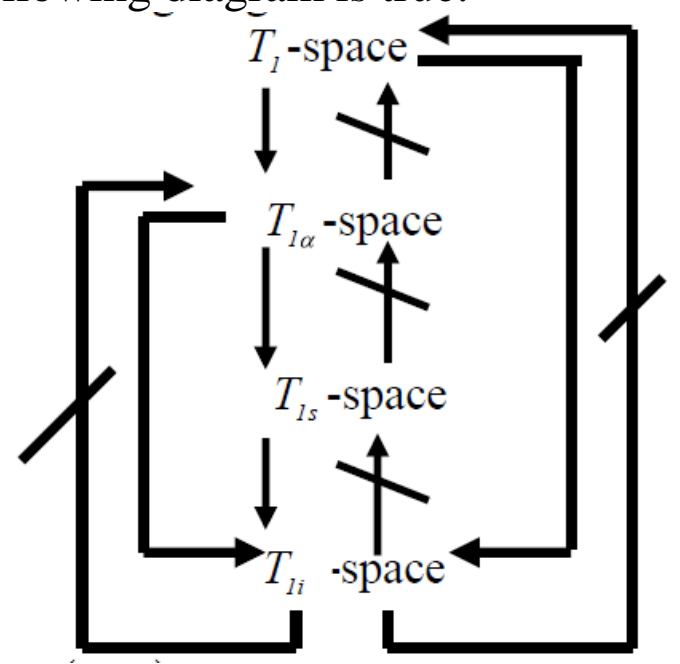

Proof: 1 . Suppose that $(X, \tau)$ is $T_{1}$-space.

Then, $\forall x, y \in X(x \neq y)$ there existstwoopen sets $I_{1}, I_{2}$ s.t. $\quad x \in I_{1}, y \notin I_{1}$, $y \in I_{2}, x \notin I_{2}$. Since every open set is $\alpha$-open (corollary1.10). Then $I_{1}$ and $I_{2}$ are $\alpha$-open sets. Therefore; $(X, \tau)$ is $T_{1 \alpha}$-space (definition 2.3).

2. Similarly, by using corollary1.10 and definition 2.3, we can prove every $T_{1 \alpha}$ - space is $T_{1 s}$ - space.

3. Similarly, by using corollary1.10 and definition 2.3 , we can prove every $\boldsymbol{T}_{1 s}$-space is $\boldsymbol{T}_{1 i}$-space.

4. From 1 and 2 we have, every $\boldsymbol{T}_{1}$-space is $\boldsymbol{T}_{1 s}$-space.

5. From 4 and 3 we have, every $\boldsymbol{T}_{1}$-space is $\boldsymbol{T}_{1 i}$ - space.

6. From 2 and 3 we have, every $\boldsymbol{T}_{1 \alpha}$-space is $\boldsymbol{T}_{1 i}$-space.

Example2.6. Let $X=\{1,2,3,4\}, \tau=\{\phi,\{1\},\{1,2\},\{1,2,3\}, X\}$, $\tau^{\alpha}=\tau^{s}=\{\phi,\{1\},\{1,2\},\{1,2,3\},\{1,3\},\{1,4\},\{1,3,4\},\{1,2,4\}, X\}$, $\tau^{i}=\{\phi,\{1\},\{1,2\},\{1,2,3\},\{2\},\{3\},\{1,2\},\{1,3\},\{2,3\}, X\}$, $(X, \tau),\left(X, \tau^{\alpha}\right),\left(X, \tau^{s}\right)$ and $\left(X, \tau^{i}\right)$ are topological -spaces.

Take, $1 \neq 2$ : 
1. There is no exists two open sets $G_{1}, G_{2}$ s.t. $l \in G_{1}, 2 \notin G_{1}, 2 \in G_{2}, l \notin G_{2}$, therefore; $(X, \tau)$ is not $T_{1}$-space.

2. There is no exists two $\alpha$-open sets $\alpha_{1}, \alpha_{2}$ s.t. $l \in \alpha_{1}, 2 \notin \alpha_{1}, 2 \in \alpha_{2}, 1 \notin \alpha_{2}$, therefore; $(X, \tau)$ is not $T_{1 \alpha}$-space.

3. There is no exists two semi-open sets $S_{l}, S_{2}$ s.t. $l \in S_{l}, 2 \notin S_{l}$, $2 \in S_{2}, l \notin S_{2}$, therefore; $(X, \tau)$ is not $T_{l s}$-space.

4. $\forall x, y \in X(x \neq y) \exists I_{1}, I_{2} \in \tau^{i}$ s.t. $x \in I_{1}, y \notin I_{1}, y \in I_{2}, x \notin I_{2}$, therefore; $(X, \tau)$ is $T_{1 i}$-space.

Definition2.7. A topological space $(X, \tau)$ is said to be $T_{2}$-space [11] (respect. $T_{2 \alpha}, T_{2 s}$ and $T_{2 i}$-space) if it satisfies Hausdorff axiom [11](respect. $\alpha$-Hausdorff, s-Hausdorff and i-Hausdorff axiom:[ $T_{2}$ (respect. $T_{2 \alpha}, T_{2 s}$ and $\left.T_{2 i}\right)$ ]: $\forall x, y \in X(x \neq y) \exists I_{1}, I_{2} \in \tau$ (respect. $\tau^{\alpha}, \tau^{s}$ and $\left.\tau^{i}\right), I_{1} \cap I_{2}=\phi \quad$ s.t. $x \in I_{1}, y \in I_{2}$.

Definition2.8. A topological space $(X, \tau)$ is said to be:

1. Regular space [11] (shortly $\mathrm{R}$ space) if it satisfies Vietoris axiom:[ $\mathrm{R}]$ if $\mathrm{F}$ is a closed set in $\mathrm{X}$ and $x \in X, x \notin F \quad \exists S_{1}, S_{2} \in \tau, S_{1} \cap S_{2}=\phi \quad$ s.t. $F \subseteq S_{1}, x \in S_{2}$.

2. $\alpha$-Regular space (shortly $\mathrm{R}_{\alpha}$-space) if it satisfies $\alpha$-Vietoris axiom: [ $\mathrm{R} \alpha$ ] if $\mathrm{F}$ is an $\alpha$-closed set in $\mathrm{X}$ and $x \in X, x \notin F \exists S_{1}, S_{2} \in \tau^{\alpha}, S_{1} \cap S_{2}=\phi$

s.t. $F \subseteq S_{1}, x \in S_{2}$.

3. s-Regular space (shortly $\mathrm{R}_{\mathrm{s}}$ space) if it satisfies s-Vietoris axiom: $\left[\mathrm{R}_{\mathrm{s}}\right]$ if $\mathrm{F}$ is a semi-closed set in $X$ and $x \in X, x \notin F \exists S_{1}, S_{2} \in \tau^{s}, S_{1} \cap S_{2}=\phi$ s.t. $F \subseteq S_{1}, x \in S_{2}$.

4. i-Regular space (shortly $R_{i}$-space) if it satisfies $\mathrm{i}$-Vietoris axiom: $\left[R_{i}\right]$ if $\mathrm{F}$ is an i-closed set in $X$ and $x \in X, x \notin F \quad \exists I_{1}, I_{2} \in \tau^{i}, I_{1} \cap I_{2}=\phi \quad$ s.t. $F \subseteq I_{1}, x \in I_{2}$.

Definition2.9. A $T_{1}$ - space[11] (respect. $T_{1 \alpha}, T_{1 s}$ and $T_{1 i}$-space) is said to be $\boldsymbol{T}_{3}$ [11] (respect. $\boldsymbol{T}_{3 \alpha}, T_{3 s}$ and $T_{3 i}$ ) if it is Regular(respect. $\alpha$-Regular, sRegular and i-Regular).

Definition2.10. A topological space $(X, \tau)$ is said to be:

1. Normal space [11] (shortly $\mathrm{N}$ space) if it satisfies Urysohn axiom: 
[N] if $F_{1} \subseteq X, F_{2} \subseteq X, F_{1} \cap F_{2}=\phi \exists S_{1}, S_{2} \subseteq X$ s.t $F_{1} \subseteq S_{1}, F_{2} \subseteq S_{2}$ where $S_{1} \cap S_{2}=\phi, F_{1}, F_{2}$ are closed sets, $S_{1}, S_{2}$ are opensets.

2. $\alpha$-Normal space (shortly $N_{\alpha}$-space) if it satisfies $\alpha$-Urysohn axiom: $\left[N_{\alpha}\right]$ if $F_{1} \subseteq X, F_{2} \subseteq X, F_{1} \cap F_{2}=\phi \exists S_{1}, S_{2} \subseteq X$ s.t $F_{1} \subseteq S_{1}, F_{2} \subseteq S_{2}$ where $S_{1} \cap S_{2}=\phi, F_{1}, F_{2}$ are $\alpha$-closed sets, $S_{1}, S_{2}$ are $\alpha$-opensets. 3. s-Normal space (shortly $N_{s}$ space) if it satisfies s-Urysohn axiom: $\left[N_{s}\right]$ if $F_{1} \subseteq X, F_{2} \subseteq X, F_{1} \cap F_{2}=\phi \exists S_{1}, S_{2} \subseteq X$ s.t $F_{l} \subseteq S_{1}, F_{2} \subseteq S_{2}$ where $S_{1} \cap S_{2}=\phi, F_{1}, F_{2}$ are semi-closed sets, $S_{1}, S_{2}$ aresemi-opensets. 4. i-Normal space (shortly $N_{i}$ space) if it satisfies i-Urysohn axiom: $\left[N_{i}\right]$ if $F_{1} \subseteq X, F_{2} \subseteq X, F_{1} \cap F_{2}=\phi \exists I_{1}, I_{2} \subseteq X$ s.t $F_{1} \subseteq I_{1}, F_{2} \subseteq I_{2}$ where $I_{1} \cap I_{2}=\phi, F_{1}, F_{2}$ arei-closedsets, $I_{1}, I_{2}$ arei-opensets.

Definition2.11. A $T_{1}$-space(respect. $T_{1 \alpha}, T_{1 s}$ and $T_{1 i}$-space) is said to be $T_{4}$ [11] (respect. $T_{4 \alpha}, T_{4 s}$ and $T_{4 i}$ if it is Normal(respect. $\alpha$ - Normal, s- Normal and i- Normal).

Definition2.12. A topological space $(X, \tau)$ is said to be:

1. Completely regular space [11] (shortly $C R$ space) if it satisfies the following axiom: $[C R]$ if $F$ is a closed set in $X$ and $x \in X, x \notin F$ there exists a continuous mapping $f: X \rightarrow[0,1]$ s.t. $f(F)=1, f(x)=0$.

2. $\alpha$-completely regular space (shortly $C R \alpha$ space) if it satisfies the following axiom: [ $C R \alpha$ ] if $\mathrm{F}$ is an $\alpha$-closed set in $\mathrm{X}$ and $x \in X, x \notin F$ there exists an $\alpha$-continuous mapping $f: X \rightarrow[0,1]$ s.t. $f(F)=1, f(x)=0$.

3. s-completely regular space (shortly $C R_{s}$ space) if it satisfies the following axiom: $\left[C R_{s}\right]$ if $F$ is a semi-closed set in $X$ and $x \in X, x \notin F$ there exists a semi-continuous mapping[5] $f: X \rightarrow[0,1]$ s.t. $f(F)=1, f(x)=0$.

4. i-completely regular space (shortly $C R_{i}$ space) if it satisfies the following axiom: $\left[C R_{i}\right]$ if $F$ is an i-closed set in $X$ and $x \in X, x \notin F$ there exist icontinuous mapping [9] $f: X \rightarrow[0,1]$ s.t. $f(F)=1, f(x)=0$.

Definition2.13. A $T_{1}$-space(respect. $T_{1 \alpha}, T_{1 s}$ and $T_{1 i}$-space) is said to be $T_{(31 / 2)}$ [11] (respect. $T_{(31 / 2) \alpha}, \quad T_{(31 / 2) s}$ and $T_{(31 / 2) i}$ ) if it is completely Regular(respect. $\alpha$ - completely Regular, s- completely Regular and icompletely Regular).

Definition2.14. A topological space $(X, \tau)$ is said to be: 
1. Completely Normal space [11] (shortly $C N$ space) if it satisfies Tietze axiom:[CN] If, $A_{1} \subseteq X, A_{2} \subseteq X, A_{1} \cap A_{2}=\phi \exists S_{1}, S_{2} \subseteq X$ s.t $A_{1} \subseteq S_{1}, A_{2} \subseteq S_{2}$ where $A_{1}, A_{2}$ aretwo separated sets, $S_{1} \cap S_{2}=\phi, S_{1}, S_{2}$ are open sets.

2. $\alpha$-completely Normal space (shortly $C N_{\alpha}$ space) if it satisfies $\alpha$-Tietze axiom: $\left[C N_{\alpha}\right]$ if, $A_{1} \subseteq X, A_{2} \subseteq X, A_{1} \cap A_{2}=\phi, \exists S_{1}, S_{2} \subseteq X$ s.t $A_{1} \subseteq S_{1}, A_{2} \subseteq S_{2}$ where $A_{1}, A_{2}$ aretwo separatedsets, $S_{1} \cap S_{2}=\phi, S_{1}, S_{2}$ are $\alpha$-opensets . 3. s-completely Normal space (shortly $C N_{s}$ space) if it satisfies s- Tietze axiom: $\left[C N_{s}\right]$ if, $A_{1} \subseteq X, A_{2} \subseteq X, A_{1} \cap A_{2}=\phi \exists S_{1}, S_{2} \subseteq X$ s.t $A_{1} \subseteq S_{1}, A_{2} \subseteq S_{2}$ where $A_{1}, A_{2}$ aretwo separatedsets, $S_{1} \cap S_{2}=\phi, S_{1}, S_{2}$ are semi-opensets. 4. i-completely Normal space (shortly $C N_{i}$ space) if it satisfies i- Tietze axiom: [ $C N_{i}$ ] if, $A_{1} \subseteq X, A_{2} \subseteq X, A_{1} \cap A_{2}=\phi \exists I_{1}, I_{2} \subseteq X$ s.t $A_{1} \subseteq I_{1}, A_{2} \subseteq I_{2}$ where $A_{1}, A_{2}$ aretwo separated sets, $I_{1} \cap I_{2}=\phi, I_{1}, I_{2}$ are $i$-opensets.

Definition2.15. A $T_{1}$ - space (respect. $T_{1 \alpha}, T_{1 s}$ and $T_{l i}$-space) is said to be $T_{5}[11]$ (respect. $T_{5 \alpha}, \quad T_{5 s}$ and $T_{5 i}$-space) if it is completely Normal(respect. $\alpha$ - completely Normal, s- completely Normal and icompletely Normal).

Example2.16. Let $X=\{a, b\}, \tau=\{\phi,\{a\},\{b\}, X\}, \tau^{\alpha}=\tau^{s}=\tau^{i}=\tau$ $(X, \tau),\left(X, \tau^{\alpha}\right),\left(X, \tau^{s}\right)$ and $\left(X, \tau^{i}\right)$ are topological spaces.

Open, $\alpha$-open, $s$-open and $i$-open sets are: $\phi,\{a\},\{b\}, X$.

Closed, $\alpha$-closed, $s$-closed and $i$-closed sets are: $\phi,\{a\},\{b\}, X$

1. $a, b \in X(a \neq b) \exists\{a\},\{b\} \in \tau\left(\right.$ respect. $\tau^{\alpha}, \tau^{s}$ and $\left.\tau^{i}\right)$ s.t. $a \in\{a\}, b \in\{b\}$. Therefore; $(X, \tau)$ is $T_{1}, T_{1 \alpha}, T_{1 s}$ and $T_{1 i}$-space.

2. $a, b \in X(a \neq b) \exists\{a\},\{b\} \in \tau\left(\right.$ respect. $\tau^{\alpha}, \tau^{s}$ and $\left.\tau^{i}\right)$ s.t. $a \in\{a\}, b \in\{b\}$, $\{a\} \cap\{b\}=\phi$. Therefore; $(X, \tau)$ is $T_{2}, T_{2 \alpha}, T_{2 s}$ and $T_{2 i}$-space.

3. $i .\{b\}$ is a closed set and $a \notin\{b\}$ there are two open sets $\{a\},\{b\}$ s.t. $a \in\{a\},\{b\} \subseteq\{b\}$. Therefore; $(X, \tau)$ is Regular space.

ii. $\{b\}$ is $\alpha$-closed set and $a \notin\{b\}$ there are two $\alpha$-open sets $\{a\},\{b\}$ s.t. $a \in\{a\},\{b\} \subseteq\{b\}$. Therefore; $(X, \tau)$ is $\alpha$-Regular space.

iii. $\{b\}$ is a semi - closed set and $a \notin\{b\}$ there are two semi-open sets $\{a\},\{b\}$ s.t. $a \in\{a\},\{b\} \subseteq\{b\}$. Therefore; $(X, \tau)$ is s-Regular space. 


\section{i-Open Sets and Separating Axioms Spaces}

$i v .\{b\}$ is an $i$-closed set and $a \notin\{b\}$ there is two $i$-open sets $\{a\},\{b\}$ s.t. $a \in\{a\},\{b\} \subseteq\{b\}$. Therefore; $(X, \tau)$ is i-Regular space.

4. By (1) and (3) (i)(respect. (ii), (iii) and (iv)) we have: $(X, \tau)$ is $T_{3}$-space (respect. $T_{3 \alpha}, T_{3 s}$ and $T_{3 i}$-space).

5. i. $\{a\},\{b\}$ are closed sets, there are two open sets $\{a\},\{b\}$

s.t. $\{a\} \subseteq\{a\},\{b\} \subseteq\{b\},\{a\} \cap\{b\}=\phi$. Therefore; $(X, \tau) \quad$ is Normal space.

ii. $\{a\},\{b\}$ are $\alpha$-closed sets, there are two $\alpha$-open sets $\{a\},\{b\}$

s.t. $\{a\} \subseteq\{a\},\{b\} \subseteq\{b\},\{a\} \cap\{b\}=\phi$. Therefore; $\quad(X, \tau)$ is $\alpha$-Normal space.

iii. $\{a\},\{b\}$ are semi-closed sets, there are two semi-open sets $\{a\},\{b\}$

s.t. $\{a\} \subseteq\{a\},\{b\} \subseteq\{b\},\{a\} \cap\{b\}=\phi$. Therefore; $\quad(X, \tau)$ is s-Normal space.

iv. $\{a\},\{b\}$ are $i$-closed sets there are two $i$-open sets $\{a\},\{b\}$

s.t. $\{a\} \subseteq\{a\},\{b\} \subseteq\{b\},\{a\} \cap\{b\}=\phi$. Therefore; $\quad(X, \tau)$ is i-Normal space.

6. By (1) and (5) (i)(respect. (ii), (iii) and (iv)) we have: $(X, \tau)$ is $T_{4}$-space (respect. $T_{4 \alpha}, T_{4 s}$ and $T_{4 i}$-space).

7. $i$. let $f: X \rightarrow[0,1]$ be a continuousmapping and $\{b\}$ is a closed set and $a \notin\{b\}$ s.t. $f(a)=0, f(\{b\})=1$.

s.t. $f(a)=0, f(\{b\})=1$. Therefore; $(X, \tau)$ is Completely Regular space.

ii. let $f: X \rightarrow[0,1]$ be an $\alpha$-continuous mapping and $\{b\}$ is $\alpha$-closed set and $a \notin\{b\}$ s.t. $f(a)=0, f(\{b\})=1$.

Therefore; $(X, \tau)$ is $\alpha$-Completely Regular space.

iii. let $f: X \rightarrow[0,1]$ be a semi-continuousmapping and $\{b\}$ is a semi-closed set and $a \notin\{b\}$ s.t. $f(a)=0, f(\{b\})=1$. Therefore; $\quad(X, \tau)$ is s-Completely

Regular space.

$i v$. Let $f: X \rightarrow[0,1]$ be an $i$-continuous mapping and $\{b\}$ isan $i$-closed set and $a \notin\{b\}$ s.t. $f(a)=0, f(\{b\})=1$. Therefore; $\quad(X, \tau) \quad$ is i-Completely Regular space.

8. By (1) and (7) (i)(respect. (ii), (iii) and (iv)) we have: $(X, \tau)$ is $T_{(31 / 2)}$ space (respect. $T_{(31 / 2) \alpha}, T_{(31 / 2) s}$ and $T_{(31 / 2) i}$-space).

9. i. $\{a\},\{b\} \subseteq X$, thereare two open $\operatorname{sets}\{a\},\{b\}$

s.t. $\{a\} \subseteq\{a\},\{b\} \subseteq\{b\}$ where $\{a\} \cap\{b\}=\phi$. 
Therefore; $(X, \tau)$ is Completely Normal space.

ii. $\{a\},\{b\} \subseteq X$, thereare two $\alpha$-open sets $\{a\},\{b\}$

s.t. $\{a\} \subseteq\{a\},\{b\} \subseteq\{b\}$ where $\{a\} \cap\{b\}=\phi$.

Therefore; $(X, \tau)$ is $\alpha$-Completely Normal space.

iii. $\{a\},\{b\} \subseteq X$, thereare two semi-open $\operatorname{sets}\{a\},\{b\}$

s.t. $\{a\} \subseteq\{a\},\{b\} \subseteq\{b\}$ where $\{a\} \cap\{b\}=\phi$.

Therefore; $(X, \tau)$ is s-Completely Normal space.

$i v .\{a\},\{b\} \subseteq X$, thereare two $i-$ open sets $\{a\},\{b\}$

s.t. $\{a\} \subseteq\{a\},\{b\} \subseteq\{b\}$ where $\{a\} \cap\{b\}=\phi$.

Therefore; $(X, \tau)$ is i-Completely Normal space.

10. By (1) and (9)(i)(respect. (ii), (iii) and (iv)) we have: $(X, \tau)$ is $T_{5}$-space (respect. $T_{5 \alpha}, T_{5 s}$ and $T_{5 i}$ - space).

Corollaries2.17. The following diagrams are true. i.

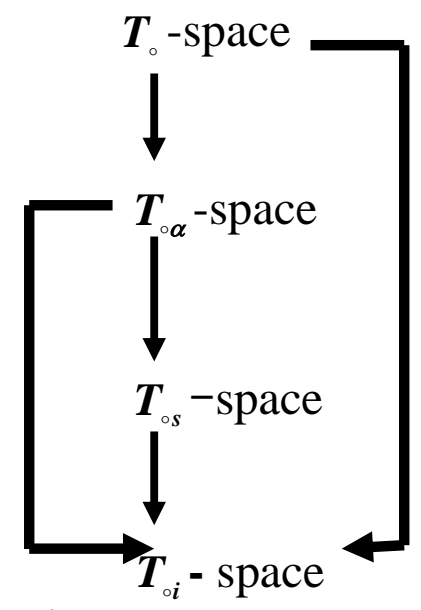

Proof: 1 . Suppose that $(X, \tau)$ is $T_{\text {。 }}$ space.

Then $\forall x, y \in X(x \neq y)$ there exists open set $I$ s.t. $x \in I, y \notin I$. Since every open set is $\alpha$-open (corollary1.10). Then $I$ is $\alpha$-open set. Therefore; $(X, \tau)$ is $T_{\text {。 }}$-space (definition 2.1).

2. Similarly, by using (corollary1.10) and (definition 2.1), we can prove every $T_{\circ \alpha}$-space is $T_{\circ s}$ - space.

3. Similarly, by using corollary 1.10 and definition 2.1 , we can prove every $T_{\text {os }}$-space is $T_{\text {oi }}$ space.

4. From 1 and 2 we have, every $T_{\circ}$-space is $T_{\text {os }}$-space.

5. From 4 and 3 we have, every $T_{\circ}$-space is $T_{\circ i}$-space. 
6. From 2 and 3 we have, every $T_{\circ \alpha}$-space is $T_{\circ i}$-space.

ii.

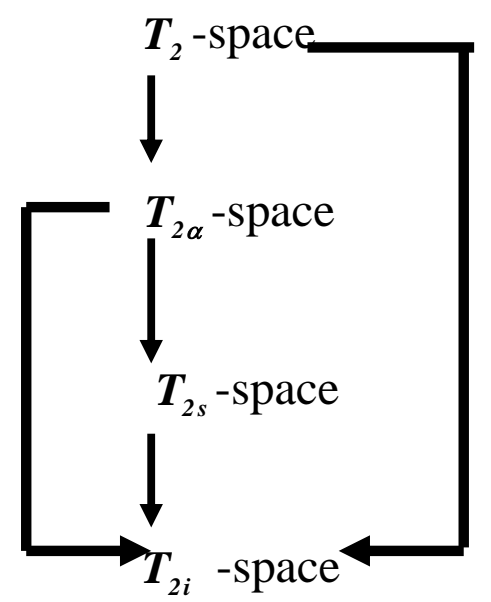

Proof: 1 . Suppose that $(X, \tau)$ is $T_{2}$-space.

Then $\forall x, y \in X(x \neq y)$ thereexiststwoopen sets $I_{1}, I_{2}, I_{1} \cap I_{2}=\phi$ s.t. $x \in I_{1}$ , $y \in I_{2}$. Since every open set is $\alpha$-open (corollary1.10). Then $I_{1}$ and $I_{2}$ are $\alpha$-open sets. Therefore; $(X, \tau)$ is $T_{2 \alpha}$ space (definition 2.7).

2. Similarly, by using (corollary1.10) and (definition 2.7), we can prove every $T_{2 \alpha}$-space is $T_{2 s}$ - space.

3. Similarly, by using corollary1.10 and (definition 2.7), we can prove every $T_{2 s}$-space is $T_{2 i}$ - space.

4. From 1 and 2 we have, every $T_{2}$ space is $T_{2 s}$ space.

5. From 4 and 3 we have, every $T_{2}$ space is $T_{2 i}$ space.

6. From 2 and 3 we have, every $T_{2 \alpha}$ space is $T_{2 i}$ space. iii.

Proof: 1 . Suppose that $(\vec{X}, \tau)$ is a regular space. Then for every closed set $F$ in $\mathrm{X}$ with $x \in X, x \notin F \quad$ thereexiststwoopen sets $S_{1}, S_{2}, S_{1} \cap S_{2}=\phi$ s.t. $F \subseteq S_{1}, x \in S_{2}$. Since every open (closed) set is $\alpha$-open $(\alpha-$ closed $)$ 
(corollary1.10).Then $S_{1}$ and $S_{2}$ are $\alpha$-open sets and $\mathrm{F}$ is $\alpha$-closed set. Therefore; $(X, \tau)$ is $\alpha$-regular space (definition 2.8(2)).

2. Similarly, by using corollary 1.10 and definitions $2.8(2), 2.8(3)$, we can prove every $\alpha$-regular space is s-regular space

3. Similarly, by using corollary 1.10 and (definitions 2.8(3), 2.8(4), we can prove every s-regular space is i-regular space.

4. From 1 and 2 we have, every regular space is s-regular space.

5. From 4 and 3 we have, every regular space is i-regular space.

6 . From 2 and 3 we have, every $\alpha$-regular space is i-regular space. iv.

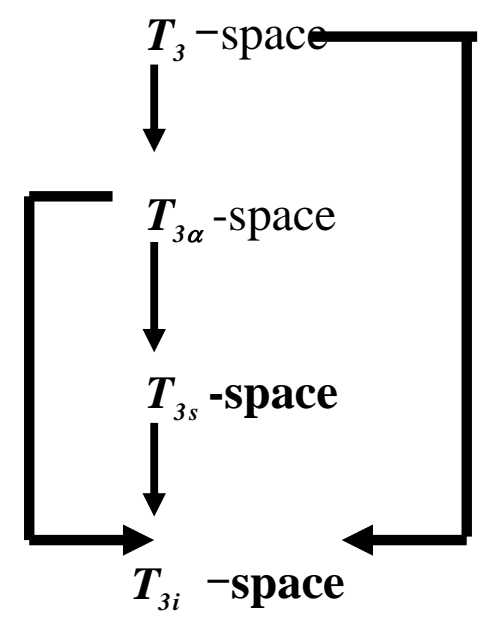

Proof: 1. Suppose that $(X, \tau)$ is $T_{3}$-space. Then $(X, \tau)$ is $T_{1}$ and regular space (definition 2.9). Since every $T_{1}$ space is $T_{l \alpha}$ (corollary $2.5(1)$ and since every Regular space is $\alpha$-Regular(corollaries 2.17(iii)(1)), we have $(X, \tau)$ is $T_{3 \alpha}$-space.

2. Similarly, by using (definition 2.8(4)), (corollary 2.5(2)) and corollaries 2.29(iii)(2), we can prove every $T_{3 \alpha}$ space is $T_{3 s}$ space.

3. Similarly, by using (definition 2.9), (corollary 2.5(3)) and corollaries 2.17(iii)(3), we can prove every $T_{3 s}$ space is $T_{3 i}$ space.

4. From 1 and 2 we have, every $T_{3}$ space is $T_{3 s}$ space.

5. From 4 and 3 we have, every $T_{3}$ space is $T_{3 i}$ space.

6. From 2 and 3 we have, every $T_{3 \alpha}$ space is $T_{3 i}$ space. 
V.

\section{Completely Regular space}<smiles>[13CH3]</smiles>

s-Completely Regular space

Proof: 1 . Suppose that $(X, \tau)$ is a completely regular space. Then for every closed set $F$ in $X$ with $x \in X, x \notin F$ there exists a continuous mapping $f: X \rightarrow[0,1]$ s.t. $f(F)=1, f(x)=0$. Since every open (closed) set is $\alpha$-open $(\alpha$-closed) (corollary1.10) and since every continuous mapping is $\alpha$-continuous(corollary 1.20). Then $\mathrm{F}$ is $\alpha$-closed set and $f: X \rightarrow[0,1]$ is $\alpha$-continuous. Therefore; $(X, \tau)$ is $\alpha$ - completely regular space (definition 2.18(2)).

2. Similarly, by using (corollary 1.10), (corollary 1.20) and (definition $2.12(3)$ ), we can prove every $\alpha$ - completely regular space is s- completely regular space.

3. Similarly, by using (corollary 1.10), (corollary 1.20) and (definition 2.12(4)), we can prove every s- completely regular space is i- completely regular space.

4. From 1 and 2 we have, every completely regular space is s- completely regular space.

5. From 4 and 3 we have, every completely regular space is i- completely regular space.

6. From 2 and 3 we have, every $\alpha$-completely regular space is icompletely regular space.

vi.
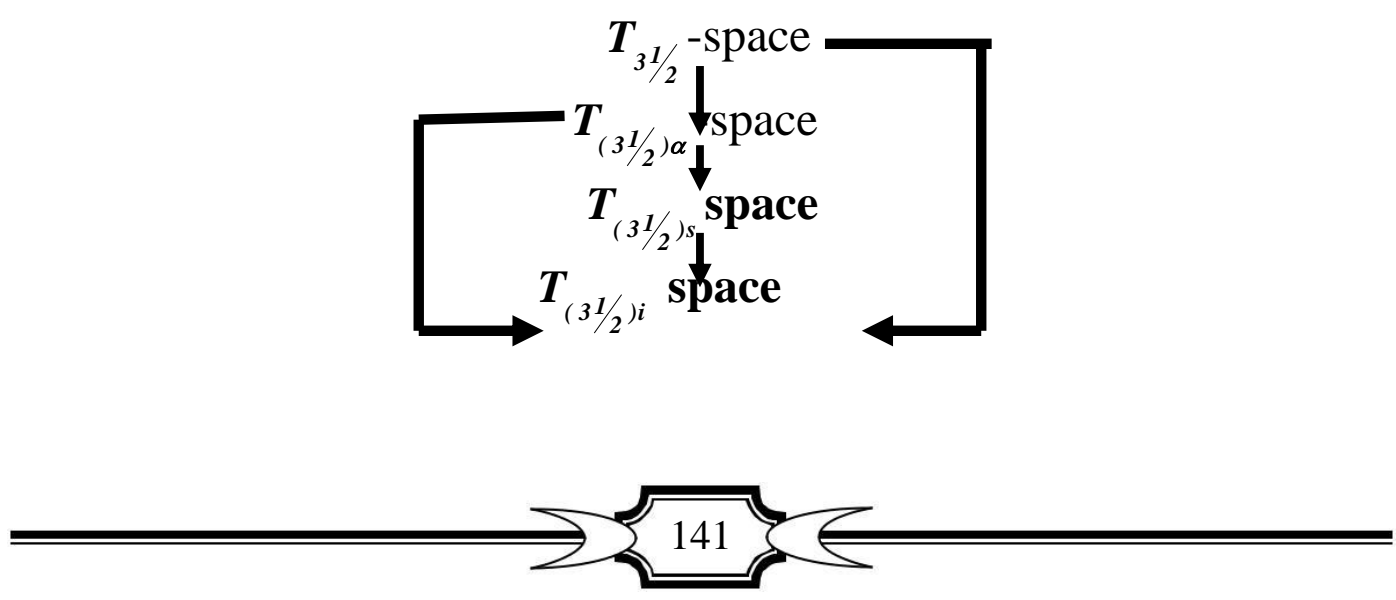
Proof: 1. Suppose that $(X, \tau)$ is $T_{31 / 2}$ space. Then $(X, \tau)$ is $T_{1}$ and completely-regular space (definition 2.13). Since every $T_{1}$ space is $T_{1 \alpha}$ (corollary 2.5(1) and since every completely regular space is $\alpha$ completely regular (corollaries $2.17(\mathrm{v})(1)$ ), we have $(X, \tau)$ is $T_{(31 / 2) \alpha}$ space.

2. Similarly, by using (corollary 2.5(2)) and corollaries $2.17(\mathrm{v})(2)$, we can prove every $T_{(31 / 2) \alpha}$ space is $T_{(31 / 2) s}$ space.

3. Similarly, by using (corollary 2.5(3)) and corollaries $2.17(\mathrm{v})(3)$, we can prove every $T_{(31 / 2) s}$ space is $T_{(31 / 2) i}$ space.

4. From 1 and 2 we have, every $T_{31 / 2}$ space is $T_{(31 / 2) s}$ space.

5. From 4 and 3 we have, every $T_{31 / 2}$ space is $T_{(31 / 2) i}$ space.

6. From 2 and 3 we have, every $T_{(31 / 2) \alpha}$ space is $T_{(31 / 2) i}$ space. vii.

Normal space
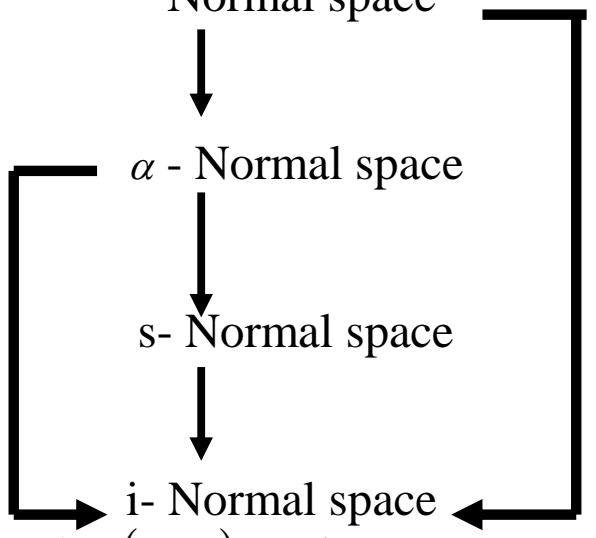

Proof: 1. Suppose that $(X, \tau)$ is a normal space. Then for every $F_{1} \subseteq X, F_{2} \subseteq X, F_{1} \cap F_{2}=\phi \exists S_{1}, S_{2} \subseteq X$ s.t $F_{1} \subseteq S_{1}, F_{2} \subseteq S_{2}$ where $S_{1} \cap S_{2}=\phi, F_{1}, F_{2}$ are closed sets, $S_{1}, S_{2}$ are opensets. Since every open (closed) set is $\alpha$-open $(\alpha-$ closed $)\left(\right.$ corollary1.10). Then $S_{1}$ and $S_{2}$ are $\alpha$-open sets and $F_{1}, F_{2}$ are $\alpha$-closed sets. Therefore; $(X, \tau)$ is $\alpha$ normal space (definition2.10 (2)).

2. Similarly, by using (corollary 1.10) and (definition 2.10(3)), we can prove every $\alpha$ - normal space is s- normal space.

3. Similarly, by using (corollary 1.10) and (definition 2.10(4)), we can prove every s- normal space is i- normal space.

4. From 1 and 2 we have, every normal space is s- normal space.

5. From 4 and 3 we have, every normal space is i- normal space.

6 . From 2 and 3 we have, every $\alpha$-normal space is i- normal space. 
viii.

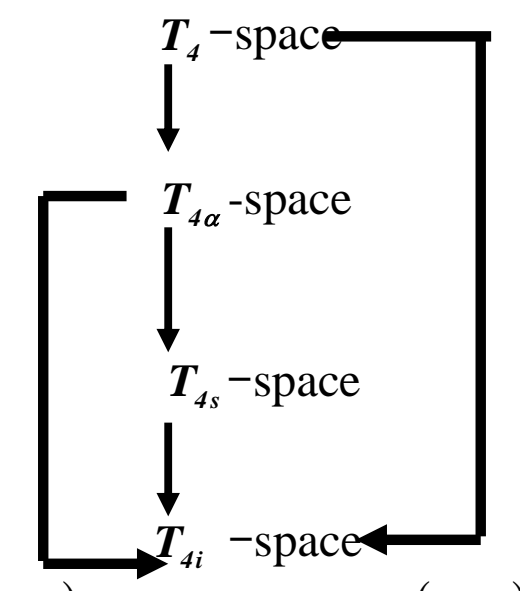

Proof: 1 . Suppose that $(X, \tau)$ is $T_{4}$ space. Then $(X, \tau)$ is $T_{1}$ and normal space (definition 2.11). Since every $T_{1}$ space is $T_{1 \alpha}$ (corollary $2.5(1)$ and since every normal space is $\alpha$-normal(corollaries 2.17 (vii)(1)), we have $(X, \tau)$ is $T_{4 \alpha}$ space.

2. Similarly, by using (definition 2.11), (corollary 2.5(2)) and corollaries 2.17(vii)(2), we can prove every $T_{4 \alpha}$-space is $T_{4 s}$ - space.

3. Similarly, by using (definition 2.11), (corollary 2.5( $($ ) ) and corollaries 2.17(vii)( $\Gamma)$, we can prove every $T_{4 s}$ - space is $T_{4 i}$-space.

4. From 1 and 2 we have, every $T_{4}$-space is $T_{4 s}$-space.

5. From 4 and 3 we have, every $T_{4}$-space is $T_{4 i}$-space.

6. From 2 and 3 we have, every $T_{4 \alpha}$ - is $T_{4 i}$-space. ix.

\section{Completely Normal space}
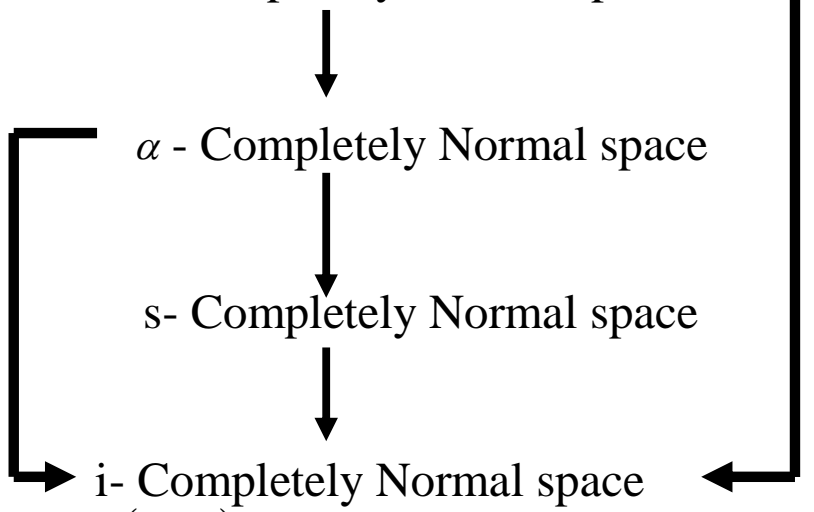

Proof: 1 . Suppose that $(X, \tau)$ is a completely normal space.

Then for every two separated subsets of $X, A_{1}, A_{2}, A_{1} \cap A_{2}=\phi$, there exists two open sets, $S_{1}, S_{2} \subseteq X$ s.t $A_{1} \subseteq S_{1}, A_{2} \subseteq S_{2}, S_{1} \cap S_{2}=\phi$. Since every 
open (closed) set is $\alpha$-open ( $\alpha$-closed) (corollary1.10). Then $S_{1}, S_{2}$ are $\alpha$-closed sets. Therefore; $(X, \tau)$ is $\alpha$ - completely normal space (definition 2.14(2)).

2. Similarly, by using (corollary 1.10) and (definition 2.14(3)), we can prove every $\alpha$-completely normal space is s- completely normal space.

3. Similarly, by using (corollary 1.10) and (definition 2.14(4)), we can prove every s- completely normal space is i- completely normal space.

4. From 1 and 2 we have, every completely normal space is s- completely normal space.

5. From 4 and 3 we have, every completely normal space is i- completely normal space.

6. From 2 and 3 we have, every $\alpha$-completely normal space is icompletely normal space.

$\mathrm{x}$.

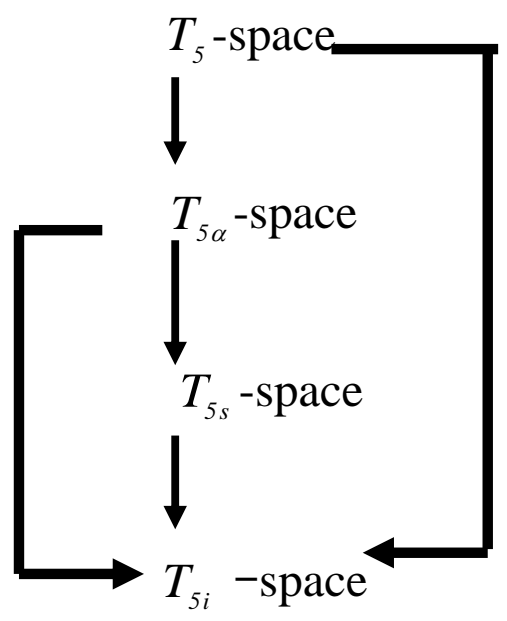

Proof: 1 . Suppose that $(X, \tau)$ is $\boldsymbol{T}_{5}$ space. Then $(X, \tau)$ is $T_{1}$ and completely normal space (definition 2.15). Since every $T_{1}$ space is $T_{1 \alpha}$ (corollary 2.5(1) and since every completely normal space is $\alpha$ - completely normal(corollaries 2.17(ix)(1)), we have $(X, \tau)$ is $T_{5 \alpha}$ space.

2. Similarly, by using (definition 2.15), (corollary 2.5(2)) and corollaries 2.17(ix)(2), we can prove every $T_{5 \alpha}$ space is $T_{5 s}$ space.

3. Similarly, by using (definition 2.15), (corollary 2.5( $\uparrow)$ ) and corollaries 2.17(ix)( $(r)$, we can prove every $T_{5 s}$ space is $T_{5 i}$ space.

4. From 1 and 2 we have, every $T_{5}$ space is $T_{5 s}$ space.

5. From 4 and 3 we have, every $T_{5}$ space is $T_{5 i}$ space.

6. From 2 and 3 we have, every $T_{5 \alpha}$ space is $T_{5 i}$ space. 
From above we have the converses of corollaries 2.17 are not necessary to be true.

\section{References}

[1]Al-Sheikhly, A.H. and Khudhair, H.K., Another Type of Separation Axioms Depend on an $\theta g$-open set, Al-Mustansiriya Univ., Journal of Education College, Vol. 1, No. 1, 2012, 66-75.

[2]Devi, R., Selvakumar, A. and Parimala, M., $\alpha \psi-$ closed sets in topological spaces, Submitted 2011.

[3]Dontchev and Maki, On $\theta$-generalized closed sets, Internet. J.math. and math. Sci. Vol. 22, No. 2, (1999), 239-249.

[4]Mohammad, Fatima, M., Pre-Techonov and Pre-Hausdorff Separation Axioms in Intuitonistic Fuzzy special topological spaces, Tikrit Journal of Pure Science Vol. 11, No. 1, 2006.

[5]Levine, N., 1963. Semi-open sets and semi-continuity in topological space, Amer. Math. Monthly 70:36-41.

[6]Levine, N., 1970. Generalized closed sets in topology. Rend. Circ. Mat. Palermo, 19(2): 89-96.

[7]Maheshwari S.N. and Prasad R., Some new separation axioms, Ann.soc.sci. Bruxelles, Ser.I.,89(1975), 395-402.

[8]Mashhour A.S., Abd El-Monsef M.E. and El-Deeb, S.N., On precontinuous and weak precontinuous mappings, proc. Math. phys. soc. Egypt, 53(1982), 47-53.

[9]Mohammed, A.A. and Askandar, S.W., On i-open sets, UAE Math Day Conference, American Univ. of Sharjah, April 14, 2012.

[10]Njastad, O., On some classes of nearly open sets, pacific J. Math. 15(1965), 961-970.

[11]Pervin, W.J., Foundations of General Topology, translated by Attallah Thamir Al-Ani, Mosul Univ., 1985.

[12]Kim, Y.K., Devi, R. and Selvakumar, A., Weakly Ultra Separation Axioms Via $\alpha \psi-$ Open sets, International Journal of Pure and Applied Mathematics, Vol. 71, No. 3, 2011, 435-440.

[13]Reilly, I.L. and Vamanmurthy M.K., on $\alpha$-continuity in topological spaces, Acta Math. Hungar., 45(1-2)(1985), 27-32. 